\title{
Correction to: Foundations of Behavioral Health
}

\author{
Bruce Lubotsky Levin and Ardis Hanson
}

\section{Correction to:
B. L. Levin, A. Hanson (eds.), Foundations of Behavioral Health, https://doi.org/10.1007/978-3-030-18435-3

1. The original version of the opening page of chapter 8 and contributors section in FM were inadvertently published with incorrect affiliation (city) information of author "Ralph J. DiClemente". This has been updated as "New York, NY, USA".

2. An email ID of the author "Kevin C. Heslin" has been also updated as "kevin heslin@gwu.edu" in chapter 2 (The Global Epidemiology of Mental and Substance Use Disorders).

3. The below mentioned content in chapter 9 has been printed twice and the duplicate occurrence has been deleted.

"While there have been some policy changes to improve health services delivery, outcomes have not been sufficient in meeting the health needs of this population. Clearly, developing amended and updated policies must address the needs of underserved populations."

\footnotetext{
The updated online versions of these chapters can be found at https://doi.org/10.1007/978-3-030-18435-3_2 https://doi.org/10.1007/978-3-030-18435-3_8 https://doi.org/10.1007/978-3-030-18435-3_9 https://doi.org/10.1007/978-3-030-18435-3
} 